\title{
COMMENT
}

\section{Inclusion of children and pregnant women in COVID-19 intervention trials}

\author{
Atul Malhotra ${ }^{1,2}$, Arunaz Kumar ${ }^{3}$, Charles C. Roehr ${ }^{4,5}$ and Maria C. den Boer ${ }^{6,7}$ \\ Pediatric Research (2021) 89:1063-1064; https://doi.org/10.1038/s41390-020-1067-3
}

The novel coronavirus (SARS-CoV-2) presents a stiff challenge for health professionals where information and research on therapeutic options for the virus and disease needs to keep abreast with clinical management of patients. There are many re-purposed and new therapies being trialed for the prevention and treatment of its clinical disease, COVID-19. A search of a trial registry (Clinicaltrials.gov) in April 2020 revealed more than 500 trials already registered for "SARS-CoV-2" or "COVID-19". A manual search of the inclusion and exclusion criteria of 250 registered interventional drug or vaccine trials revealed that more than $80 \%$ trials exclude children or pregnant women. On one hand, pregnant women are at a similar risk of contracting the virus as the general population, and their risk of adverse outcomes from COVID-19 is not thought to be high. ${ }^{1}$ On the other hand, how the virus, the disease or treatment may affect the fetus and the neonate is not yet entirely understood. Similarly, there are only a limited numbers of trials, which include children. Children of all ages are planned to be included in the RECOVERY trial, a national trial of COVID-19 conducted from Oxford University in the United Kingdom (https://www.recoverytrial.net/). Some other trials include children over 12 years or 15 years of age, but the inclusion of children is more an exception to the rule instead of the norm for COVID-19 intervention trials. Although severe COVID19 in children seems rare, adverse outcomes in children, such as respiratory or multiorgan failure, acute respiratory distress syndrome (ARDS), and even death have been reported. ${ }^{2}$ We wonder whether it is justified that so many ongoing or planned trials exclude children and pregnant women. Can we afford to wait for intervention trials to be completed on the adult, nonpregnant population to make a judgement on the use of COVID19 candidate drugs in children and pregnant women with suspected or proven COVID-19 or should we involve them in trials now?

\section{PREGNANT WOMEN AND COVID-19 INTERVENTION TRIALS}

Pregnant women have historically been excluded from clinical trials of new drugs or therapies for the general adult population. ${ }^{3}$ In some ongoing and planned trials for COVID-19, a prerequisite for inclusion of female adult participants is for them to practice birth control or use a medically accepted contraceptive method. When such a requisite has been put forward in the past, those trials ran the risk of inadvertent pregnancies and exposure to trial drugs in an uncontrolled manner. ${ }^{4}$ The exclusion of pregnant participants was traditionally justified due to the unforeseen teratogenic risks to the fetus and adverse pregnancy outcomes of new medications. However, it has been argued that excluding pregnant women from research participation can only be justified if the trial has no prospect of medical benefit to the pregnant woman, and the studied intervention has a known or plausibly inferred risk of significant harm to the fetus. ${ }^{2}$ Although longstanding consensus suggests that the inclusion of pregnant women in research should be promoted, the recruitment and retention of pregnant women in interventional trials of adult population continues to remain a challenge. ${ }^{5}$

\section{CHILDREN AND COVID-19 INTERVENTION TRIALS}

Just as is the case in pregnant women, children have historically been excluded from the vast majority of intervention trials. ${ }^{6}$ Rightly so, children are seen as a vulnerable population that needs to be protected against undue risks from research, and children are therefore often excluded from research. ${ }^{7}$ However, children are not simply small adults and imputation of information gained from adult studies to the pediatric population is scientifically and ethically wrong. Pediatric physiology, disease processes, and the pharmacokinetics of drugs used are likely to be different in children. Thus, excluding children from research trials therefore poses them at potential risk of harm through off-label or compassionate use of medications or interventions.

To date, almost all COVID-19 intervention trials exclude children. However, like pregnant women, children affected with COVID-19 can have emergency access to drugs like remdesivir through compassionate grounds, but not in the setting of a designed clinical trial. ${ }^{8}$ Similarly despite its unproven efficacy in any population, the emergency use of hydroxychloroquine for adolescents who weigh $50 \mathrm{~kg}$ or more can be authorized ${ }^{9}$ in clinical settings but not in research. In all these situations, children affected with COVID-19 are likely to be exposed to the potential risks of harm through these drugs, but may lack the benefits of properly conducted research. Instead, recruiting children in these trials can provide grounds for justified use of medications in future, thereby, supporting evidence-based practice.

\section{DISCUSSION}

A recent example of excluding children and pregnant women in drug and vaccine trials was the Ebola epidemic in Africa. ${ }^{10}$ On

\footnotetext{
Monash Newborn, Monash Children's Hospital, Melbourne, VIC, Australia; ${ }^{2}$ Department of Paediatrics, Monash University, Melbourne, VIC, Australia; ${ }^{3}$ Department of Obstetrics and Gynaecology, Monash University, Melbourne, VIC, Australia; ${ }^{4}$ National Perinatal Epidemiology Unit, Nuffield Department of Population Health, Medical Sciences Division,

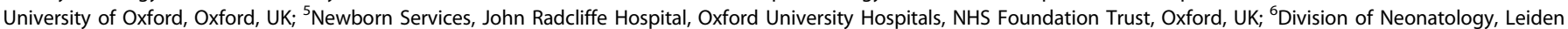
University Medical Center, Leiden, The Netherlands and ${ }^{7}$ Department of Medical Ethics and Health Law, Leiden University Medical Center, Leiden, The Netherlands Correspondence: Atul Malhotra (atul.malhotra@monash.edu)
}

Received: 22 April 2020 Revised: 4 May 2020 Accepted: 1 July 2020

Published online: 20 July 2020 
multiple occasions across a number of trials in that epidemic, opportunities were lost for children, pregnant and lactating women and their infants ${ }^{11}$ to receive potentially life-saving interventions. These were available to the rest of the adult population, and if women and children had been included it would have helped to obtain vital research data for the this cohort for future use. We argue that making the same mistake for COVID19 can, and should be, avoided and that the inclusion of children and pregnant women in trials of COVID-19 should be promoted.

In order to guarantee the ethical conduct of clinical research, various guidelines are available. One of the key principles is that clinical research should have a favorable risk-benefit ratio. Estimating the risk-benefit ratio for intervention trials of COVID19 is complex, due to the unpredictability related to the behavior of the novel virus, the disease or its treatment on fetuses, neonates and children. However, the anticipated delay in time for completion of animal-reproduction studies or studies in the adult, non-pregnant population on therapeutic options is not practical. This uncertainty may explain why so many trials of COVID-19 exclude children and pregnant women a priori. We argue that this is questionable, and possibly, undesirable. By doing so, interventional trials of COVID-19 may not fairly benefit all people. Potential beneficial effects and life-saving interventions may be denied to children and pregnant women, especially, in case of serious adverse outcomes such as severe pneumonia or ARDS. Furthermore, by excluding them from these trials, children and pregnant women are put at risk of potential harm through off-label and compassionate use of non-evidence-based interventions. Moreover, ethical research and clinical practice respects the autonomy of pregnant women and mature minors to make their own informed decisions about consent and research participation.

In this pandemic, we invite researchers and institutional review boards to be transparent about their reasons for excluding children and pregnant women from intervention trials of COVID19. Doing so may allow for a scientific debate about when the inclusion of children and pregnant women in these trials is appropriate, thus promoting the inclusion of children and pregnant women in intervention trials of COVID-19. Furthermore, with SARS-CoV-2 and its clinical disease COVID-19 affecting the daily life of so many people around the world, this unprecedented time of human disruption may provide valuable opportunities for community engagement, providing insight into how children and pregnant women estimate the benefit-risk ratio of trials of COVID-19 in times of uncertainty. Moreover, community engagement will allow us to identify communication strategies to inform children and pregnant women about the benefits, risks, and uncertainties of trials of COVID-19. This will give children and pregnant women the opportunity to provide their truly informed consent.
COVID-19 presents us with a unique public health scenario, an unusual fortuity, where scientists, researchers, health administrators, health-care providers, and institutional review boards can work closely together with children and pregnant women, to discover the best treatment options in evidence-based care. Otherwise, we risk missing this rare and incidental opportunity of being inclusive in our approach, to provide equitable and just care and generate robust evidence for the management of this vital portion of the population.

\section{AUTHOR CONTRIBUTIONS}

All authors contributed to the manuscript and were involved in the concept and design of manuscript. A.M. wrote the first draft, and approved the final manuscript as submitted. All other authors added to the various sections of the manuscript and approved the final manuscript.

\section{ADDITIONAL INFORMATION}

Competing interests: The authors declare no competing interests.

Publisher's note Springer Nature remains neutral with regard to jurisdictional claims in published maps and institutional affiliations.

\section{REFERENCES}

1. Dotters-Katz, S. K. \& Hughes, B. L. Considerations for obstetric care during the COVID-19 pandemic. Am. J. Perinatol. 37, 773-779 (2020).

2. Ludvigsson, J. F. Systematic review of COVID-19 in children shows milder cases and a better prognosis than adults. Acta Paediatr. 109,1088-1095 (2020).

3. Blehar, M. C. et al. Enrolling pregnant women: issues in clinical research. Women's Health Issues 23, e39-e45 (2013).

4. Nicolas, P. et al. Safety of oral ivermectin during pregnancy: a systematic review and meta-analysis. Lancet Glob. Health 8, e92-e100 (2020).

5. FDA. Pregnant women: scientific and ethical considerations for inclusion in clinical trials. https://www.fda.gov/regulatory-information/search-fda-guidancedocuments/pregnant-women-scientific-and-ethical-considerations-inclusionclinical-trials (2018).

6. Spriggs, M. \& Caldwell, P. H. The ethics of paediatric research. J. Paediatrics Child Health 47, 664-667 (2011).

7. ICTRP. Clinical trials in children. https://www.who.int/ictrp/child/en/ (2020).

8. Stories@Gilead. An open letter from our Chairman and CEO. https://www.gilead. com/stories/articles/an-open-letter-from-our-chairman-and-ceo (2020).

9. FDA. Fact sheet for health care providers. https://www.fda.gov/media/136537/ download (2020).

10. Gomes, M. F., de la Fuente-Nunez, V., Saxena, A. \& Kuesel, A. C. Protected to death: systematic exclusion of pregnant women from Ebola virus disease trials. Reprod. Health 14(Suppl 3), 172 (2017).

11. Schwartz, D. A. Clinical trials and administration of Zika virus vaccine in pregnant women: lessons (that should have been) learned from excluding immunization with the Ebola vaccine during pregnancy and lactation. Vaccines (Basel) 6, 81 (2018). 